\title{
DIVISION OF LABOR WITHIN THE WORKER CASTE \\ OF FORMICA PERPILOSA WHEELER (HYMENOPTERA: FORMICIDAE)*
}

\author{
By Carlos Roberto F. Brandão \\ Museu de Zoologia, Universidade de São Paulo \\ São Paulo, Brasil
}

\section{INTRODUCTION}

Polymorphism, in the study of social insects, is defined as the existence within an individual colony of two or more phases or castes belonging to the same sex, without particular regard to their genetic or environmental origin (Wilson, 1953). The adaptive result of the development of female polymorphism is the division of labor within the colony. In most ants this division is clearly seen between reproductive and non-reproductive caste, but less evident within the worker caste (Oster \& Wilson, 1978). The present article utilizes the Fagen \& Goldman (1977) method for estimating the total repertory size of behavioral categories of each worker subcaste listed on an ethogram or behavioral catalog.

Ethograms are the essential first step of the comparative study of behavior (Wilson, 1974). A behavioral catalog of Formica perpilosa Wheeler, a weakly polymorphic species of the neogagates group (Buren, 1968), was constructed in order to investigate behavioral differences between the major size groups, defined here arbitrarily as three worker subcastes. Formica perpilosa is a common ant in the southern United States and northern Mexico (Gregg, 1963). It feeds mainly on plant exudates and tends membracids of the genus Pubilia (LaBerge, 1952). Its physiology has been relatively well studied by Schumacher \& Whitford (1974), Kay \& Whitford (1975), Whitford, Kay \& Schumacher (1975) and Schumacher \& Whitford (1976). The genus Formica is of unusual interest because its species are either monomorphic or weakly polymorphic and thus span the early stage of caste evolution. Yet close studies of the polymorphic species have not been undertaken.

*Manuscript received by the editor October 10, 1978. 


\section{MATERIAL AND METhodS}

Founding queens were collected near Portal, Arizona, in May, 1975 , by B. Hölldobler and individually placed in test tubes, $14.8 \mathrm{~cm}$ long by $23 \mathrm{~mm}$ inner diameter, kept moist by compact cotton plugs that trap water at the bottom of the tubes. As the selected colony grew the tube was moved to a plexiglass box $28 \mathrm{~cm} \times 45 \mathrm{~cm}$ and $15 \mathrm{~cm}$ deep, the sides of which were coated with Fluon GP-1 (Northeast Chemicals Co., Woonsockett, R.I.) to prevent escape, and four similar tubes added. The colony has since been maintained on an artificial diet for ants (Bhatkar \& Whitcomb, 1970) and honey water three times a week and fed freshly killed cockroaches (Nauphoeta cinerea) once a week.

Before the first set of observations the colony was moved to a glass nest made of two square plates $12.5 \mathrm{~cm}$ on a side, held apart by small pieces of non-toxic plasticin and taped on three sides. This simple nest permits close observation of the behavior of the entire colony. The assemblage was put on the floor of the original container next to the water tubes. The remainder of the floor served as foraging space and was kept clear for observation.

The container was small enough to be placed under a swinging arm dissecting microscope. During a period of 4 weeks, a total of 18 hours were dedicated to cataloging behavior; 2809 separate behavioral acts were recorded. The observation hours ranged randomly from 9:00 A.M. to 11:00 P.M.; no differences in level or pattern of activity were noted, related to time of day.

This species is polymorphic in the sense of Wilson (1935); for the purpose of this investigation the workers were classified in 3 groups, minors, medias and majors. Samples of 15 specimens of each group were selected later for head width measurements in order to check the adequacy of the classification. Specimens that could not be readily placed in one of the size classes were not included in the ethogram.

\section{RESULTS}

The behavioral catalog of Formica perpilosa is presented on Table 1. Twenty-eight behavioral categories were observed in the minor category, 34 in the media, and 11 in the major. By fitting the frequency data to a lognormal Poisson distribution (Fagen \& Goldman, 1977), the total numbers of categories, including those 
Table 1. Relative frequencies of behavioral acts by the three worker categories of a single colony of Formica perpilosa. (N, total number of behavioral acts recorded for each caste). Approximate population of the nest: one nest queen, 150 minor workers, 270 media workers, 30 major workers, 50 eggs, 7 larvae and 5 pupae.

Behavioral Acts

A-Grooming

1-Autogrooming head, 1st pair of legs

2-Autogrooming 2 nd pair of legs

3-Autogrooming abdomen, 3rd pair of legs

4-Allogrooming minor workers

5 -Allogrooming media workers

6-Allogrooming major workers

7-Allogrooming nest queen

B-Brood Care

8-Standing at the brood pile

9-Carry egg (or eggs in succession)

10-Lick egg (or eggs in succession)

11 - Carry larva (or larvae in succession)

12-Feed larva

13-Lick larva (or larva in succession)

14-Carry pupa

15-Lick pupa

16-Assist eclosion to adult

C-Regurgitation Behavior

17-Regurgitation with minors

18 - Regurgitation with medias

19-Regurgitation with majors

20-Donating to the Queen

21-Lay trophic egg

22 -Feed queen trophic egg

23-Feed larva trophic egg

24-Carry infrabucal pellet

25 - Feed on infrabucal pellet

D-Working

26-Foraging outside the nest

27-Feed on diet

28 -Feed on honey

29-Feed on cockroaches

30-Carry live nestmate

31-Carry dead nestmate

32-Drag the nest Queen

33 - Handle nest material

34-Carry debris

35 - Lick nest wall

36-Excavating

E-Other Behaviors

37-Antennal tipping

38-Jittering

Total

$\begin{array}{ccc}\text { minor } & \text { media } & \text { major } \\ \text { workers } & \text { workers } & \text { workers } \\ \mathbf{N}=996 & \mathrm{~N}=1679 & \mathrm{~N}=134\end{array}$

.167

.131

.062

.224

.060

.022

.019

.095

.012

.049

.030

.043
.007

.011

.077

.055

.053

.024

.004

.031

0

02

.002

.024

.070

.040

.007

0

0

0

0

.003

.007

.024

.165

.021

.009

0

.001

.001

.009

.011

.082

.022

.029

.020

0

0

0

.018

.033

.059

.092

.021

.004

.025

.007

.001

.003

.001

.005

.003

.034

.012

.001

.016

.002
0

1.0

1.0

0

0

0

0

0

0

0

0

0

0

0
0

0

0

no. behavioral categories observed per caste

$28 \quad 34$ 
not seen, were estimated to be respectively 29,36 and 11 , with 95 per cent confidence intervals of $(25,33)(25,47)$ and $(5,19)$.

\section{COMMENTS ON TABLE 1:}

\section{Sorne behavioral categories deserve special mention:}

1. Carry pupa or lick pupa: Care of pupae consisted exclusively of assisting eclosion to adult. The pupae remained in the brood chamber and only when the colony was disturbed (which did not happen during the drawing of the catalog) media workers carried them to the new nest.

2. Lay trophic egg: The actual laying of trophic eggs was not seen, but 3 times media workers were seen offering the larvae and the queen small, round, shiny objects, looking like eggs, but different from the normal ones laid by the nest queen. It is not impossible that the laying of trophic eggs is a real but rare event.

3. Recruitment behavior: In order to determine whether the workers utilize any kind of food recruitment, the colony was deprived of food for one week and the nest connected to an arena by a bridge of round sticks. Honey water was presented on the arena floor. Two periods of one hour observation were recorded. Initially all ants that reached the food source were collected and not allowed to return by the bridge. Between the two experiments the honey source, the bridge and the arena floor were changed to avoid recognition by the ants. In the first period 35 ants reached the food source, in the second 262. This experiment clearly shows that this species uses food recruitment. Returning ants were observed to rub the tips of the abdomen on the bridge sticks (probably laying a scent trail) and to display no nestmates. During the construction of the catalog, however, no recruitment behavior was noted, probably because the colony was kept fed to saturation.

4. Jittering and antennal tipping: These behavioral categories were described by Wilson (1976), but nothing is known about their meaning.

5. Defensive behavior: Our perpilosa colony was not stressed to ellicit defensive behavior. However, a stray individual of the ant Novomessor cockerelli was found on the nest floor being attacked by media and minor workers of Formica perpilosa; not even in this situation the majors were observed outside the nest. This of course does not mean that majors cannot play a role in defending the nest against predators or raids by other ants, but it is apparent defense is not their characteristic behavior. A more detailed account of the defensive behavior of this species should be useful.

After the behavioral catalog was drawn, 50 specimens had their head widths and lengths measured and plotted. The linear anamorphosis afforded a linear regression of head length on head width, where the coefficient of determination is $\mathrm{r}^{2}=.964$. When logarithmically plotted, in order to compare with Wilson's model 
(1953), the log-log anamorphosis afforded a linear regression of head length on head width. The equation is

or, alternatively,

$$
\log \mathrm{L}=.2795-.8602 \log \mathrm{W}
$$

$$
\mathrm{L}=1.9033 \mathrm{~W}^{.8602}
$$

The coefficient of determination, $\mathrm{r}^{2}$ is also .964 , showing of course an excellent fit.

I have chosen the following criteria for the definition of the categories of workers: minors, head width $1 \mathrm{~mm}$ or less; medias, 1 to $1.4 \mathrm{~mm}$; majors, larger than $1.4 \mathrm{~mm}$.

The majors represent only $6.6 \%$ of the nest population and their behavioral catalog comprises merely 11 behavioral categories. Their behavior, as seen in Table 2, is mostly directed toward grooming and regurgitation with nestmates.

\section{DisCUSSION AND CONCLUSION}

The behavioral catalog and the head shape curve of our Formica perpilosa colony show that the worker caste is strongly polyethic, in spite of being only slightly polymorphic. The media category is responsible for most of the behavioral categories. The majors are specialized in regurgitation with nestmates and may act as a trophic subcaste as in Camponotus (Colobopsis) fraxinicola (Wilson, 1974). $F$. perpilosa majors also possess a relatively large abdomen and

Table 2. Relative frequencies of groups of behavioral acts listed on Table 1 by the three worker subcastes in a single colony of Formica perpilosa. (N. total number of behavioral acts recorded for each subcaste.)

behavioral acts

$$
\text { Groups of }
$$

(from Table 1)

A-Grooming

B-Brood Care

C-Regurgitation

D-"Working"

E-Jittering \& Antennal tipping

$\begin{array}{ccc}\begin{array}{c}\text { minor } \\ \text { workers }\end{array} & \begin{array}{c}\text { media } \\ \text { workers }\end{array} & \begin{array}{c}\text { major } \\ \text { workers }\end{array} \\ \mathrm{N}=996 & \mathrm{~N}=1679 & \mathrm{~N}=134 \\ .365 & .390 & .380 \\ .137 & .248 & .134 \\ .127 & .241 & .486 \\ .355 & .116 & 0 \\ .017 & .002 & 0\end{array}$


were never observed performing any defensive or "working" behavior. They were recorded as using almost half of their time doing trophallaxis or offering infrabucal pellets to minors and medias.

King and Walters (1950) have found a very similar situation in "Formica rufa melanotica" ( $=F$. obscuripes) in natural nests. They were able to prove a correlation between polymorphism and polyethism, showing that minor workers were specialized in attending aphids. The major workers again remained inside the nest most of the time.

On Table 2 one can notice that all the categories of $F$. perpilosa spend the same amount of time performing grooming behavior. The minors are almost exclusively responsible for several tasks grouped as "working" and the medias spend a more uniform amount of time performing each group of behavioral acts.

Wilson (1953) proposed a model for the origin and evolution of polymorphism in ants. In 1971 he reviewed the ant caste system and said that five steps can be recognized in the evolution within the worker caste: monomorphism, monophasic allometry, diphasic allometry, triphasic allometry and complete dimorphism.

Age polyethism is the responsible phenomenon for caste structure in monomorphic species, and the polyethic classes are sometimes referred to as physiological classes. Traniello (1978) reported an apparent lack of temporal division of labor in the primitive ponerine ant Amblyopone pallipes, which appears to have the most primitive caste system yet documented in ants.

Monophasic allometry is the commonest manifestation of nonisometric growth. Studies on the division of labor in weakly polymorphic species showed a "by preponderance" division of labor, i.e., any given worker should be capable of performing any given normal task.

In Formica polyctena size variation is weakly correlated with division of labor. The behavioral variation observed by Otto (1958) consisted mainly of age polyethism and individual peculiarities. Although the $F$. perpilosa subcastes were not divided using age parameters, I believe that age does not account for a large fraction of the total behavioral variation.

Majors of advanced polymorphic ant species, especially completely dimorphic species, where intermediates no longer exist and the two remaining classes are remarkably different in morphology, 
usually act as soldiers. This is not true in the present case, where another kind of specialization was revealed.

In the complete dimorphic species there is no behavioral overlap between the castes. Each category can be easily distinguished from the others morphologically and behaviorally.

Majors of Zacryptocerus varians (members of one of the nine genera in which complete dimorphism is easily recognized) function primarily in defense (Wilson, 1976) and deserve the title of soldiers. Yet they sometimes wash and manipulate larvae and pupae. But during a high intensity attack, majors block the nest entrance with their saucer-shaped heads and are more persistent and effective than minors in forcing the enemy back to the entrance and finally out of the nest.

Dr. Edward O. Wilson, reviewing the manuscript, was kind enough to suggest that $F$. perpilosa majors represent an early stage in the evolution toward repletes, as seen in Proformica and Myrmecocystus (Wilson, 1971). The specialization shown by perpilosa majors (see Table 2) agrees with this view.

$F$. perpilosa seems to occupy, from the viewpoint of evolution of division of labor, an intermediary position between monomorphic and completely dimorphic species.

\section{ACKNOWLEDGMENTS}

I am indebted to the "Fundação de Amparo à Pesquisa do Estado de São Paulo" for a scholarship (Biológicas 77/1208). I wish to express my appreciation to my parents for supporting my visit to Harvard University; and to Prof. and Mrs. B. Patterson, Dr. and Mrs. W. L. Brown, Jr., and Dr. E. E. Williams for their kind hospitality.

I am specially grateful to Dr. E. O. Wilson for the opportunity to learn ant rearing techniques, for the suggestion of the problem, assistance and uses of facilities during the research and critically reviewing the manuscript. I thank Dr. and Mrs. B. Hölldobler, Gary Albert, Hiltrud Engels and specially Katherine Horton and James Traniello for advice and help with the ethogram method, and also the latter for showing me his unpublished data on Amplyopone behavior; Mr. O. Schmidt for conducting the repertory estimations; Dr. Francisca C. do Val for critically reading the manuscript. 
Finally, I am grateful to Dr. P. E. Vanzolini for the helpful suggestions during the elaboration of this paper.

\section{REFERENCES}

BHATKAR, A., AND W. H. Whitcomb.

1970. Artificial diet for rearing various species of ants. Florida Entomol. 53: 229-232.

BUREN, W.F.

1968. Some fundamental taxonomic problems in Formica (Hymenoptera: Formicidae). J. Georgia Entomol. Soc., 3(2): 25-40.

Fagen, R. M., AND R. N. Goldman.

1977. Behavioral Catalogue Analysis Method. Anim. Behav., 25(2): 261-274. GREGG, R. E.

1963. The ants of Colorado. University of Colorado Press, Boulder, Colorado. $\mathrm{xvi}+792 \mathrm{pp}$.

Kay, C. A., AND W. G. Whitford.

1975. Influences of temperature and humidity on oxygen consumption of five Chihuahuan desert ants. Comp. Biochern. Psysiol. A., 52: 281-286.

KING, R. L., AND F. WALTERS.

1950. Population of a colony of Formica rufa melanotica Emery. Proc. Iowa Acad. Sci., 57: 469-473.

LABERge, W. E.

1952. Locality records of two ants found in Kansas. J. Kansas Entornol. Soc. 25: 59 .

Oster, G. E., AND E. O. WiLson.

1978. Cast and Ecology in the Social Insects. Princeton University Press, Princeton, New Jersey (in press).

Oтто, D.

1958. Über die Arbeitsteilung im Staate von Formica rufapratensis minor Gösswald und ihre. Verhaltensphysiologischen Grundlagen: Ein Beitrag zur Biologie der Roten Waldameise. Wiss. Abh. Deutsche Akad. Landw. - Wiss Berlin 30: 1-169.

Schumacher, A. M., AND W. G. Whitford.

1974. The foraging ecology of two species of Chihuahuan desert ants: Formica perpilosa and Trachymyrmex neomexicanus (Hymenoptera: Formicidae). Ins. Soc. 21: 317-330.

1976. Spatial and temporal variation in Chihuahuan desert ant faunas. Southwest Natur. 21: 1-8.

Traniello, J. F. A.

1978. Caste in a primitive ant: Absence of age polyethism in Amblyopone. Unpublished manuscript.

Whitford, W. G., C. A. AND A. M Schumacher.

1975. Water loss in Chihuahuan desert ants. Physiol. Zool. 48: 390-397. 
WILSON, E. O.

1953. The origin and evolution of polymorphism in ants. Quat. Rev. Biol. 28: 136-156.

1971. The Insect Societies. Belknap Press of the Harvard University Press, Cambridge, Massachusetts, ix $+548 \mathrm{pp}$.

1974. The soldier of the ant Camponotus (Colobopsis) fraxinicola as a trophic caste. Psyche 81(1): 182-188.

1976. A social ethogram of the neotropical arboreal ant Zacryptocerus varians (Fr. Smith). Anim. Behav. 24(2): 354-363. 

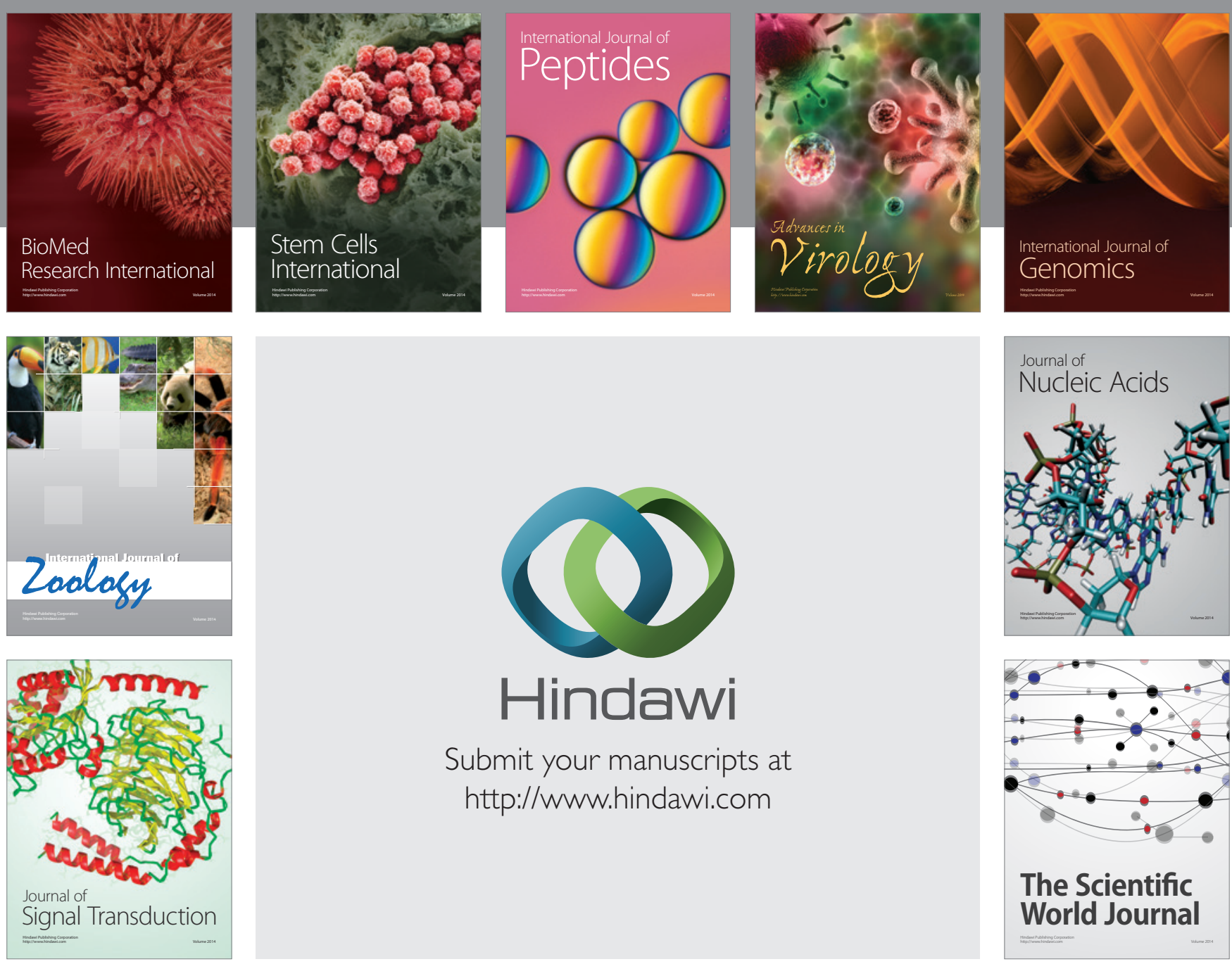

Submit your manuscripts at

http://www.hindawi.com
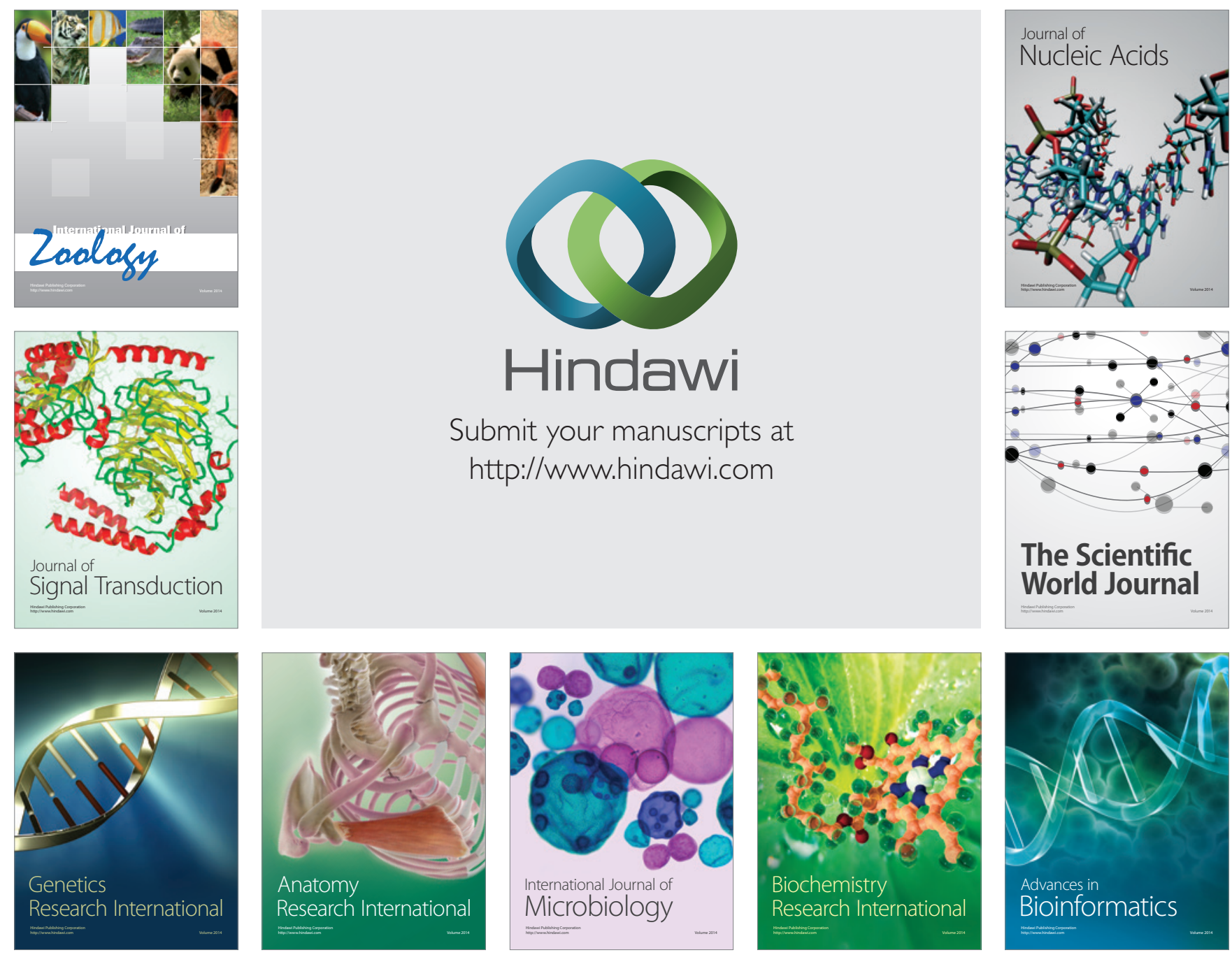

The Scientific World Journal
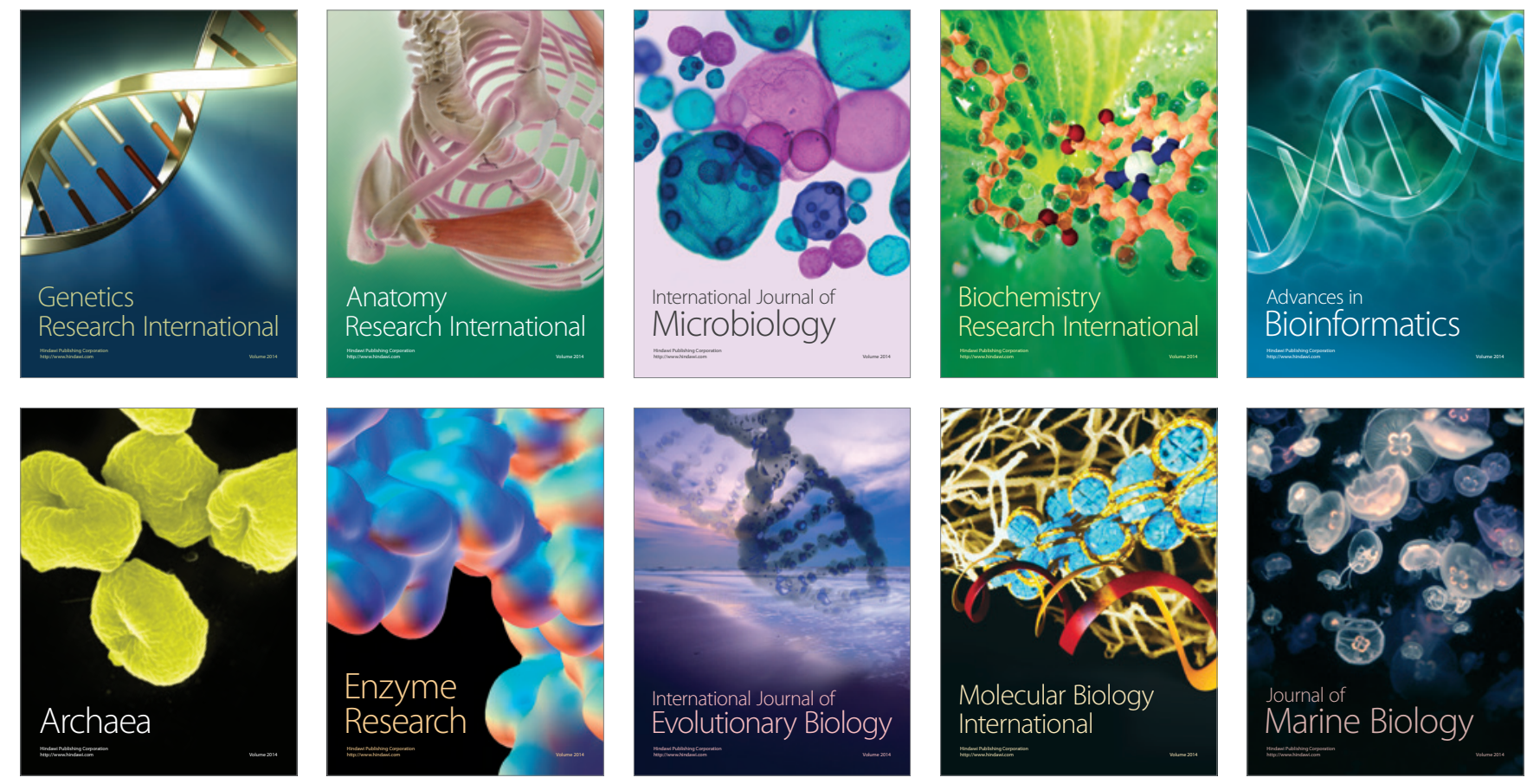